\title{
Nanomaterials for subsurface application: study of particles retention in porous media
}

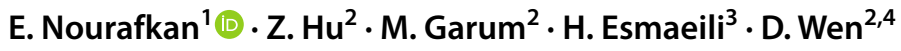

Received: 18 January 2021 / Accepted: 15 April 2021 / Published online: 7 May 2021

(c) The Author(s) 2021

\begin{abstract}
The ability to transport nanoparticles through porous media has interesting engineering applications, notably in reservoir capacity exploration and soil remediation. A series of core-flooding experiments were conducted for quantitative analysis of functionalized $\mathrm{TiO}_{2}$ nanoparticles transport through various porous media including calcite, dolomite, silica, and limestone rocks. The adsorption of surfactants on the rock surface and nanoparticle retention in pore walls were evaluated by chemical oxygen demand (COD) and UV-Vis spectroscopy. By applying $\mathrm{TiO}_{2}$ nanoparticles, 49.3 and $68.0 \mathrm{wt}$ \% of surfactant adsorption reduction were observed in pore walls of dolomite and silica rock, respectively. Not surprisingly, the value of nanoparticle deposition for dolomite and silica rocks was near zero, implying that surfactant adsorption is proportional to nanoparticle deposition. On the other hand, surfactant adsorption was increased for other types of rock in presence of nanoparticles. 5.5, 13.5, and $22.4 \mathrm{wt} . \%$ of nanoparticle deposition was estimated for calcite, black and red limestone, respectively. By making a connection between physicochemical rock properties and nanoparticle deposition rates, we concluded that the surface roughness of rock has a significant influence on mechanical trapping and deposition of nanoparticles in pore-throats.
\end{abstract}

Keywords Surface chemistry $\cdot$ Nanoparticle deposition $\cdot$ Porous media $\cdot$ Surfactant adsorption

\section{Introduction}

Nowadays, nanotechnology has become one of the promising approaches in enhanced hydrocarbon recovery, soil remediation and reservoir characterization. 0D (e.g. QDs), 1D (e.g. CNTs), 2D (e.g. Graphene oxide) and 3D NPs (e.g. silica, titanium oxides and alumina) were successfully applied for reservoir exploration (Hu et al. 2019), foam stabilizing (Yekeen et al. 2017) and enhanced oil recovery (EOR) (Luo et al. 2016; Haruna et al. 2019). Due to the relatively small

E. Nourafkan

enorafkan@gmail.com

$\triangle$ D. Wen

d.wen@leeds.ac.uk; d.wen@buaa.edu.cn

1 School of Engineering and Materials Science, University of Queen Mary, London, UK

2 School of Chemical and Process Engineering, University of Leeds, Leeds, UK

3 Department of Chemical Engineering, Bushehr Branch, Islamic Azad University, Tehran, Iran

4 School of Aeronautic Science and Engineering, Beihang University, Beijing, China size of NPs, they are much more sensitive to the physical and chemical heterogeneities present in the subsurface. Transport of bare or functionalized NPs through saturated porous media under different ionic strength conditions was a topic of several researches (Babakhani et al. 2017; Qin et al. 2020; Foroozesh and Kumar 2020). Bayat et al. (2014; 2015a, b, c) studied the transport of bare metal oxide NPs $\left(\mathrm{Al}_{2} \mathrm{O}_{3}\right.$ and $\mathrm{TiO}_{2} \mathrm{NPs}$ ) through limestone including kaolinite, montmorillonite, and illite (clay minerals). They concluded that the recovery of NPs in effluent solution had noticeably declined in the presence of clay minerals which was attributed to the trapping of NPs in pore-throats and morphology of the clays.

The NPs flooding is more effective than water flooding for subsurface applications but much less than chemical flooding. Therefore injection of NPs along with low salinity water (LSW) or chemicals (surfactant/polymer) is suggested to alter the rheological properties of injecting fluid, reduce IFT between oil/aqueous phases and decrease the surfactant adsorption on the pore walls of porous media (Olayiwola and Dejam 2019; Venancio et al. 2020). Surfactant slugs could decrease the interfacial tension (IFT) between oil and aqueous phases which results in reducing the fluid capillary force in pore-scale, mobilizing more residual crude oil in 
pore structures. Furthermore, surfactants help to maintain NPs integrity in harsh subsurface conditions of reservoirs (Nourafkan et al. 2018a, b, 2019). Employing the synergistic effect between NPs and surfactant is a promising idea for the improvement of chemical flooding efficiency (Fig. 1) (Wu et al. 2017; De Avila et al. 2016; Venancio et al. 2020). Yekeen et al (2019) studied the amount of adsorption of different surfactants (CTAB, SDBS, Triton X-100) on Malaysia shale rock in the presence and absence of $\mathrm{SiO}_{2} \mathrm{NPs}$. Maximum $49.83 \%$ and $81.33 \%$ reduction in the adsorbed surfactant on pore walls was reported at $3 \mathrm{wt} \% \mathrm{NaCl}$ salinity and high temperature $\left(80^{\circ} \mathrm{C}\right)$, respectively. Venancio et al. (2020) showed that surface modification of silica NPs with alkyl groups (octyl and hexadecyl) increased the surfactant (SDS) recovery after nanofluid injection in an unconsolidated porous medium. The reason was due to the additional hydrophobic interaction between NPs and surfactant tails which improved the colloidal stability of NPs as compared

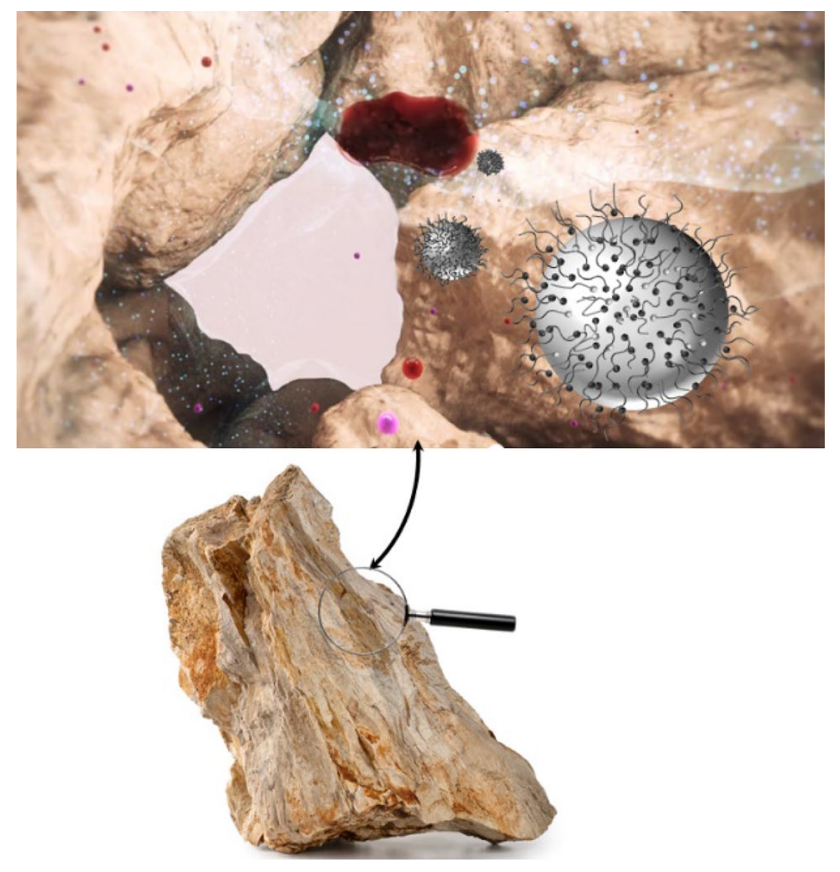

Fig. 1 Employing the synergistic effect between NPs and surfactant for chemical flooding to bare silica NPs when dispersed in micellar solutions of SDS (Venancio et al. 2020). Betancur et al. (2019) evaluated the impact of magnetic NPs on the adsorption reduction of surfactants mixture (propoxy sulfate and olefin sulfonate) in the sand pack porous media.

Although several researches have been done for the evaluation of functionalized NPs transport through porous media, but there is no consensus among researchers regarding the effect of rock properties on the efficiency of NPs transport. It has been long recognized that the mineralogy and surface structure of rocks significantly could affect the efficiency of NPs and chemical flooding (Arsalan et al. 2015; Liang et al. 2020). Therefore, the effect of physicochemical properties of porous media on the retention of functionalized NPs in pore walls must be considered while designing the process. This study aims to address the gap in the literature by investigating the effect of pore wall's properties on the transport of surfactant and functionalized NPs through five different types of reservoir rocks. The main important achievement of this study is that the efficiency of chemical flooding could significantly improve by adding NPs into flooding process specifically for reservoir rock with dense smooth surface (here silica and dolomite).

\section{Experimental procedure}

\section{Materials and characterization}

Anionic alkyl aryl sulfonic acid (AAS), nonionic alcohol ethoxylated (EA, C12-13/7EO) surfactants and Titanium (IV) oxide NPs were used as model formulation (surfactants mixture as chemical agents and NPs as carrier). Five different types of reservoir rock were crushed and sieved. The particle size fraction of $250-425 \mu \mathrm{m}$ was selected, washed three times with de-ionized water, and decanted to remove all dust particles. Then the rock grains were put inside an oven at $80^{\circ} \mathrm{C}$ for 5 days to dry and remove residual humidity to be ready for the BET analysis. A piece of dry rock was polished using different grades of sandpaper (including very-fine sandpaper size) to smooth the rock surface (Fig. 2). The polished and cleaned rock pieces were then washed with
Fig. 2 The polished rock surfaces for contact angle measurement

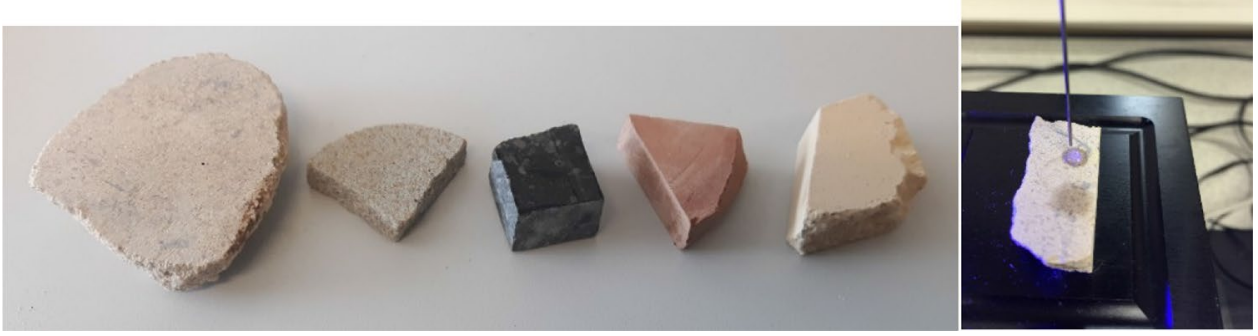


water and dried in an oven. A water droplet (usually 1-10 $\mu$ l) was dispensed on top of rock pieces using a $0.74 \mathrm{~mm}$ outer diameter syringe needle and contact angle was calculated using a goniometer right after (CAM 2008, KSV instruments Ltd. Finland).

The surface chemistry and elemental analysis of rocks were analyzed using an SEM-EDEX analysis. The EDX analysis was performed for specific points or defined areas on sample surfaces for elemental analysis. Moreover, the Zeta potential and hydrodynamic size of rock particle as well as $\mathrm{TiO}_{2} \mathrm{NPs}$ were measured in brine by Malvern Zeta-sizer ZS instrument.

\section{Core-flooding tests}

Figure 3 shows the schematic of core-flooding set-up. The brine and nanofluid slugs were injected into the core holder using a peristaltic pump and a syringe pump, respectively. The core-holder was filled with $10 \mathrm{~g}$ of different rock particles (250-425 micron) and all flooding tests were carried out at ambient temperature $\left(22^{\circ} \mathrm{C}\right)$. The permeability value in the range of $90-110 \mathrm{mD}$ was calculated based on Darcy's law using the average pressure gradient at both ends of the packed bed column during brine saturation (Supplementary document). The NP's concentration in the effluent at the outlet of core-holder was measured using UV-spectrophotometer at a wavelength of $450 \mathrm{~nm}$ (Shimadzu, UV 1800). The calibration curve of $\mathrm{TiO}_{2}$ NPs concentration versus UV absorption ratio (at wavelength of $450 \mathrm{~nm}$ ) was generated with a series of standard samples. The concentration of $\mathrm{TiO}_{2}$ NPs in effluent solution was estimated using UV intensity by interpolation from the calibration curve. On the other hand, the stabilizer's concentration was specified by chemical oxygen demand (COD). Core-flooding experiments were carried out as follows:

- $100 \mathrm{ml}$ brine flooding at a flow rate of $2 \mathrm{ml} / \mathrm{min}$ to saturate the rock particles in the core-holder.

- $20 \mathrm{ml}$ surfactant slug or nanofluid (functionalized NPs with surfactants) at a flow rate of $0.5 \mathrm{ml} / \mathrm{min}$.

- $20 \mathrm{ml}$ brine post-flooding at a flow rate of $1 \mathrm{ml} / \mathrm{min}$.

\section{Results and discussion}

\section{Characterization of physical and chemical properties of different rocks}

Limestone is a sedimentary rock, which composes a large amount of calcium carbonate mineral with some variable amounts of silica. According to SEM photos (Fig. 4) the grains of limestone are irregularly shaped and well-crystallized grains are very rare. Elemental mapping of limestone rocks (Fig. S1) shows the existence of manganese and iron in both limestones. The black limestone contains a higher amount of magnesium oxide mineral, which is the main reason of black color. Goethite or hematite is the probable mineralogy of iron in the red limestone as previously investigated by Cai et al. (2012); however, the existence of hematite is the reason for red color in limestone rock. The elemental mapping also verifies the existence of clay minerals (Alumina and silica composition) in the limestone rocks.

The SEM and EDX images of other types of rocks have been provided in the supplementary document. The SEM photos of the calcite rock show the existence of OOlits structure inside the carbonate rock (Fig. S2). The vein of silica mineral is also detected in calcite rock (Fig. S2) while the
Fig. 3 Schematic of core-flooding set-up

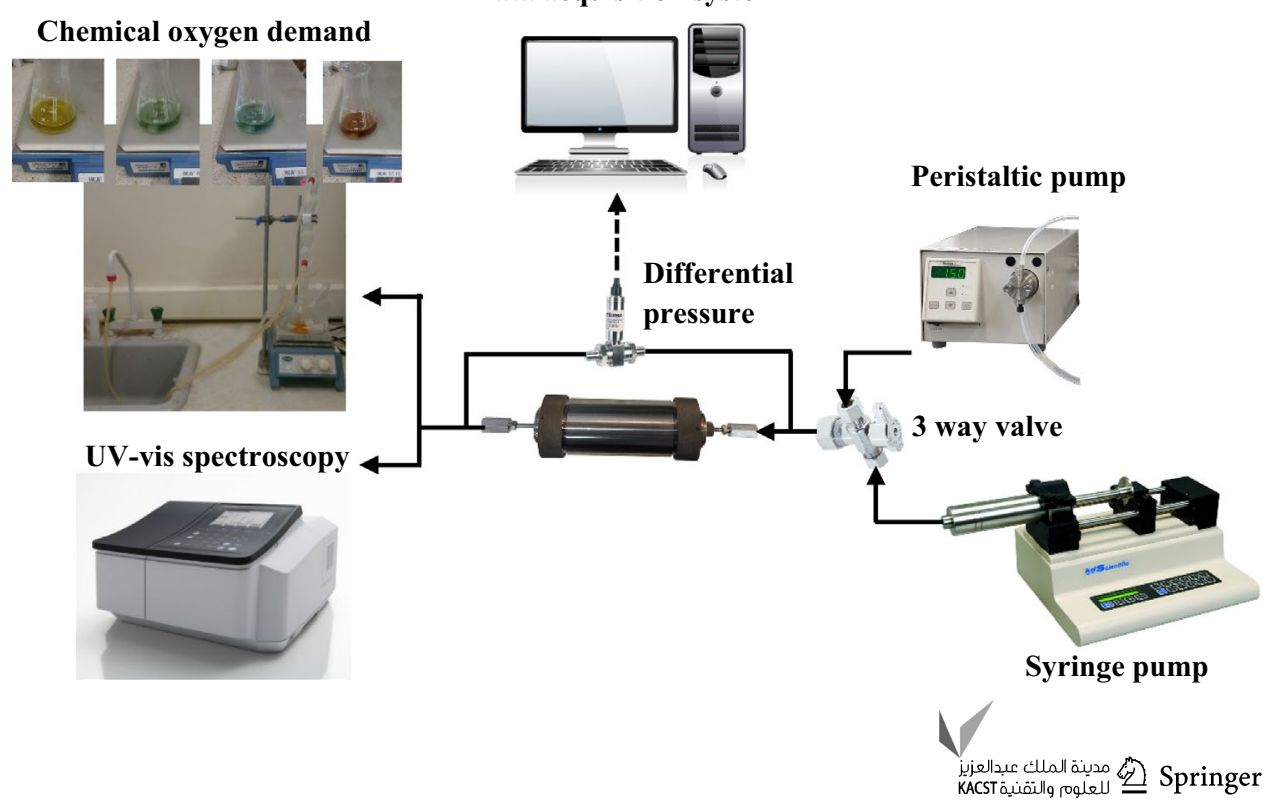


Fig. 4 SEM photos of a black limestone and $\mathbf{b}$ red limestone
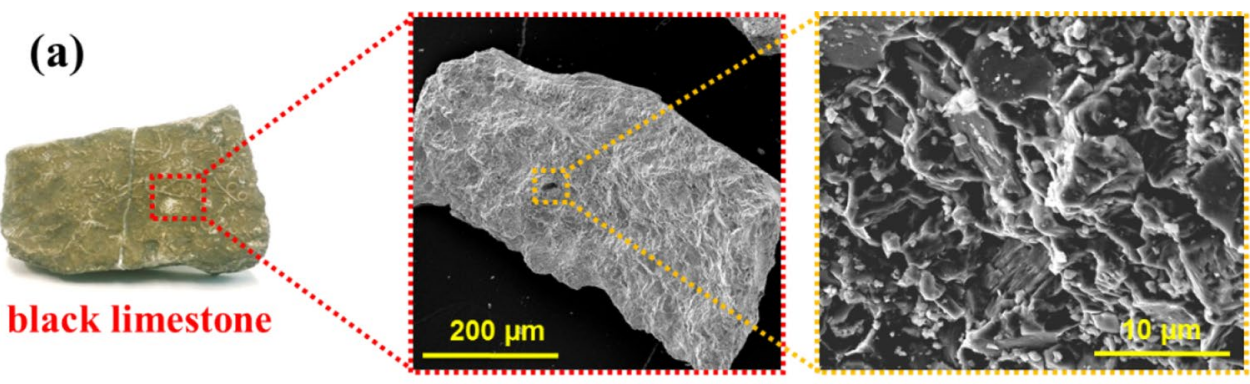

(b)
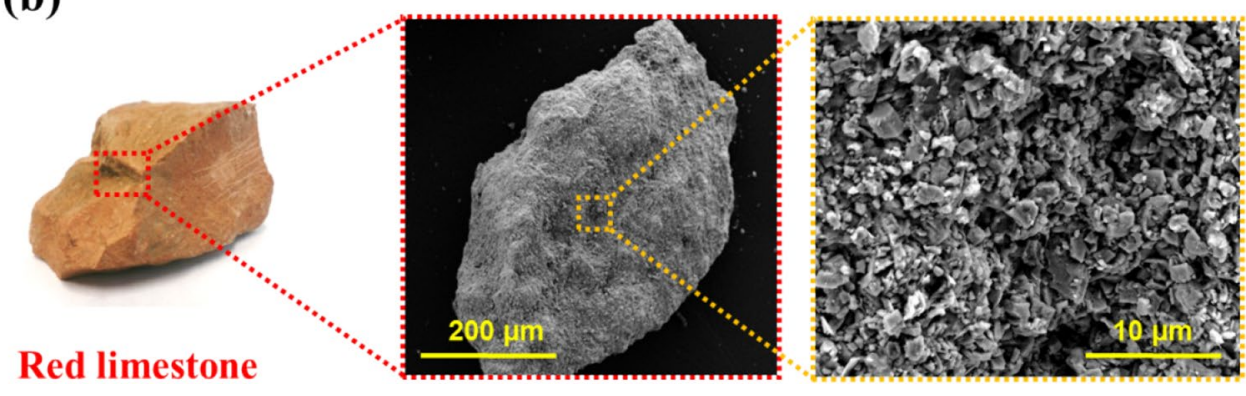

composition of dolomite substrate was uniform including magnesium and calcium (Fig. S3). Furthermore, the elemental map of silica rock (Fig. S4) confirms that the silicate crystals is the major fraction of rock structure which combined with a minor fraction of Aluminosilicate mineral.

The surface charge of different rocks was obtained using electrophoresis measurements of crushed rock (Schramm et al. 1991). Rock particles smaller than 45 microns were separated using sieve analysis and suspended in deionized water followed by a high-speed centrifuge (5000 RPM for half an hour). The electrophoretic mobility of the final suspension then was measured by Malvern zetasizer (Table 1). According to Table 1 all rocks have negative surface charges with the following trend:

Dolomite (least negative) $<$ calcite $<$ black limestone $<$ red limestone $<$ Silica (most negative).

Quantitative measurement of CA can determine the wettability of a rock. According to the CA's data (Table 1, Fig. S5) the black limestone and silica have the lowest and highest degree of water wettability between rocks. Surface roughness, fluid composition, and rock mineralogy are the most important factors which affect the CA of rocks. First time, Wenzel (1936) investigated the effect of roughness on $\mathrm{CA}$ and proposed a relationship for the angle observed on both smooth and rough surfaces. Vijapurapu et al. (2002) also studied the impact of mineralogy and surface roughness on the wettability of different rocks including quartz, berea sandstone, dolomite, and calcite. Both studied reported that the CA values strongly depend on surface chemistry and roughness. However, the Wenzel equation did not match the observation of Vijapurapu.

\section{Functionalizing of $\mathrm{TiO}_{2} \mathrm{NPs}$ with stabilizers}

The AAS surfactant solution samples ( $15 \mathrm{ml}, 0.3 \mathrm{wt} \%)$ at different salinities have been shown in Fig. S6. According to Fig. S6 the AAS surfactant made a cloudy solution or even is not soluble at salinity higher than $2 \mathrm{wt} \%$. Clarity and long-term stability are important factors in the design of an injectable surfactant slug. A cloudy and unstable slug indicates an ineffective surfactant solution formulation in the desired salinity range. Thus, it will be necessary to find a suitable composition for formulating single-phase aqueous surfactant solutions at different salinities. Using additive

Table 1 The physical and chemical properties of different rocks

\begin{tabular}{llllll}
\hline Rock type & Silica & Black limestone & Red limestone & Dolomite & Calcite \\
\hline Specific surface area $\left(\mathrm{m}^{2} / \mathrm{g}\right)$ & $0.877 \pm 0.001$ & $1.884 \pm 0.003$ & $3.09 \pm 0.004$ & $2.54 \pm 0.021$ & $1.46 \pm 0.0013$ \\
Electrophoretic mobility & $-1.58 \pm 0.09$ & $-1.07 \pm 0.07$ & $-1.14 \pm 0.04$ & $-0.83 \pm 0.05$ & $-1.00 \pm 0.07$ \\
$\quad\left(\mu \mathrm{m} \mathrm{cm} \mathrm{V} \mathrm{s} \mathrm{s}^{-1}\right)$ & -20.6 & -14.2 & -15.2 & -11.1 & -12.1 \\
Zeta potential $(\mathrm{mV})$ & 20.09 & 68.23 & 37.88 & 29.83 & 40.89 \\
Contact angle & & & &
\end{tabular}


(such as a minor fraction of oil phase) or blend of surfactant can produce a suitable aqueous solution for injection. In this study, EA surfactant was added to AAS surfactant solution. The ethylene oxide groups in the structure of EA provide tolerance to salinity which produced a clear aqueous solution. In fact, the blending of AAS with EA promotes the resistance of micelles over a high salinity environment and shifts the unsolvable surfactants to become more hydrophilic results in the formation of a clear aqueous solution.

Critical micelle concentration (CMC) of AAS was determined by surface tension measurement of surfactant solution with air and conductivity method (Fig. 5). The conductivity of the solution was measured by a Mettler Toledo conductivity Meter (Seven2Go). The conductivity of anionic surfactant solution increased linearly with increasing surfactant concentration until it reached $2.4 \times 10^{-3} \mathrm{~g} / \mathrm{ml}$, beyond which the rate of conductivity increasing was slightly reduced. This point on the graph where the slope of the conductivity line was changed has been identified as the CMC. Therefore, the selected surfactant concentration for the EOR process could be sensible, considering the $\mathrm{CMC}$ value.

$\mathrm{TiO}_{2}$ NPs were selected in this study as an example carrier for surfactant molecules in porous media. The optimum condition of functionalizing (concentration of $\mathrm{TiO}_{2} \mathrm{NPs}$, salinity, and optimum surfactants ratio) and formation of non-covalent grafting surfactants on NPs of solution were evaluated in our previous study (Nourafkan et al. 2018b). Briefly, $\mathrm{TiO}_{2}$ suspensions were prepared by homogenizing $2000 \mathrm{ppm}$ of $\mathrm{TiO}_{2}$ nanopowders inside the 25\% AAS-75\% EA surfactants blend solution (4 wt $\%$ salinity) by an ultrasound probe running $15 \mathrm{~min}$ with an amplitude of 25. The reason for choosing $2000 \mathrm{ppm}$ for NPs concentration has been provided in the supplementary document. The stability of nanofluid was checked

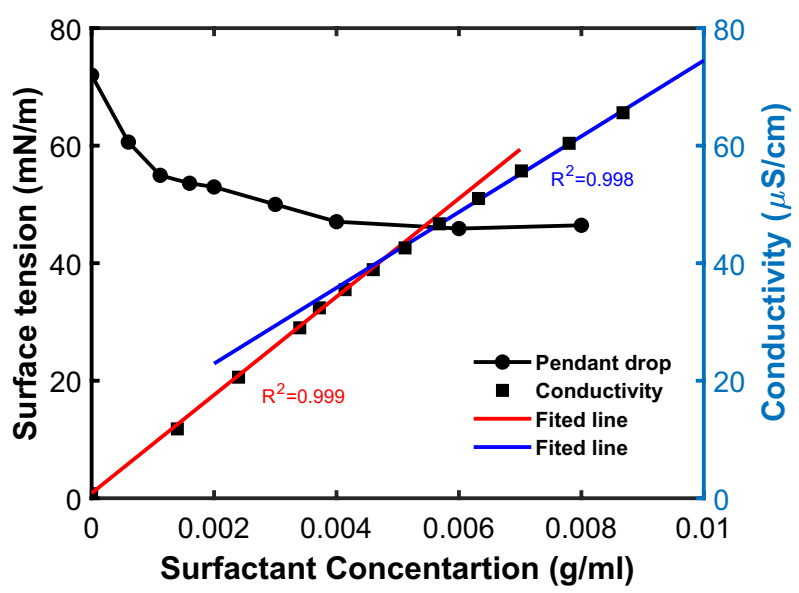

Fig. 5 Critical micelle concentration of AAS surfactant using surface tension measurement of surfactant solution with air and conductivity method by UV-visible method and no change was observed for adsorption peak of nanofluid after $1.5 \mathrm{~h}$ immobility. The zeta potential and hydrodynamic size of $\mathrm{TiO}_{2} \mathrm{NPs}$ in brine ( 4 wt. $\%$ salinity, neutral $\mathrm{pH})$ were $-10.1(\mathrm{mV})$ and $147 \mathrm{~nm}$ respectively. The breakthrough curves (BTCs) of NPs were generated using on-line measurement of concentration data using UV-Vis analysis. So, the calculated concentrations in the effluent stream divided by the initial concentration of NPs were drawn versus the injection time expressed in pore volumes (PVs). Finally, the deposition of NPs ( $\mathrm{mg} / \mathrm{g}$ rock and $\mathrm{mg} / \mathrm{m}^{2}$ rock) then calculated by mass balance calculation using BTCs. The breakthrough curves (BTCs) of $\mathrm{TiO}_{2} \mathrm{NP}$, which shows relative adsorption, as a function of PV are provided in Fig. 6. The intensity of spectra generally decreases by passing time, which shows that significant amounts of particles were exited from porous media during the flooding stage. However, the amount of NPs deposition rate (average intensity of spectral data) are different for different rocks. For example, the average intensity of black limestone and calcite packs is lower compared to silica and dolomite packs which show more retention of NPs in these porous media. The intensity results of post brine flooding (blue points in Fig. 6) show that driving out of NPs is continued following by the brine flooding. However, after totally $20 \mathrm{PV}$, no more NPs can be cleaned out.

Figure $7 \mathrm{a}$ and Table 2 represented total weight percent of $\mathrm{TiO}_{2}$ NPs which trapped in porous media and those discharged during flooding and post-flooding which were calculated from BTCs.

Figure 7a and Table 2 indicate that all NPs were discharged from silica and dolomite porous media while other rocks have a high degree of NPs retention. Caldelas et al. (2011) stated that the specific surface area of rock has a linear effect on particle retention independently of lithology. However, in this study, the surface area of dolomite rock is relatively high in comparison to other rocks (Table 1) while NPs were completely recovered through porous media of dolomite. Guzman et al. (2006) also stated that the surface charge of rocks is a primary factor in the retention of $\mathrm{TiO}_{2}$ NPs in porous media. Different mechanisms of adsorption, gravitational sedimentation, interception, straining and mechanical trapping have been suggested for the deposition of NPs during transport through porous media, Fig. 8 (Medjda et al. 2020; Agista et al. 2019).

During this sedimentation process, the particles having greater solid density than water settle on the rock surface. Due to the high stability of NPs, sedimentation is unlikely to be the main mechanism for NPs deposition in the current study. Moreover, both Brownian motion and the short residence time of NPs could disable gravity sedimentation in the porous media. Independency of NPs deposition amount to the surface charge of the rocks could imply that

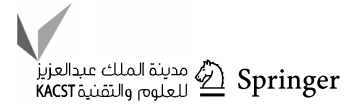



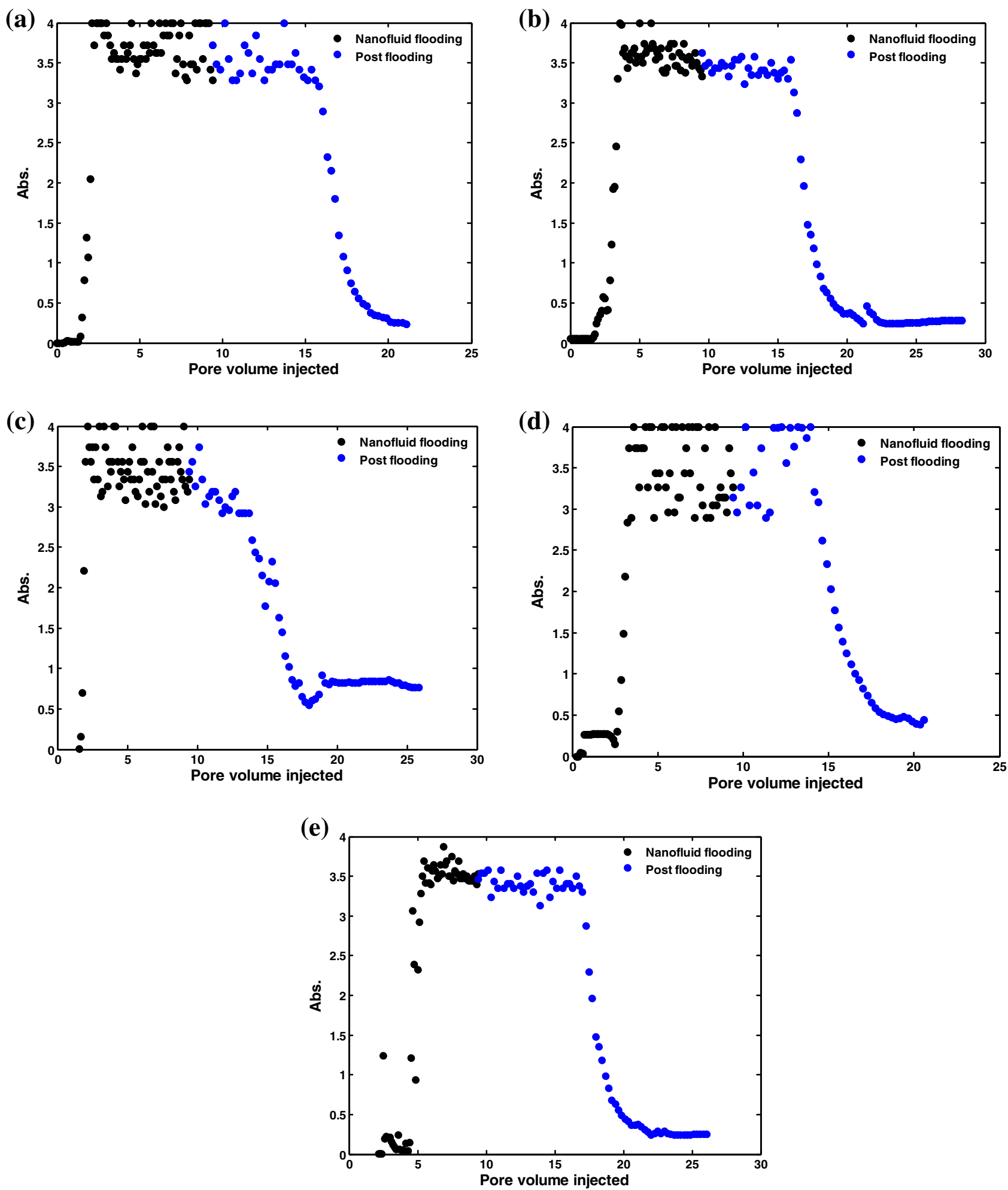

Fig. $6 \mathrm{TiO}_{2}$ NPs breakthrough curves transported through different rocks: a silica, b black limestone, $\mathbf{c}$ red limestone, $\mathbf{d}$ dolomite, e Calcite

the adsorption does not play the main role in trapping the particles. Moreover, the hydrocarbon tail of surfactants does not let the NPs adsorb on the surface of water-wet rocks.
Straining is defined as any physical trapping of agglomerated NPs in pore-throats narrower than the size of larger particles after agglomeration (Babakhani et al. 2017). The 
Fig. 7 a Weight percent of trapped NPs in porous media, $\mathbf{b}$ adsorbed surfactants blend (25\% AAS-75\% EA) on rock surface with and without NPs (a)

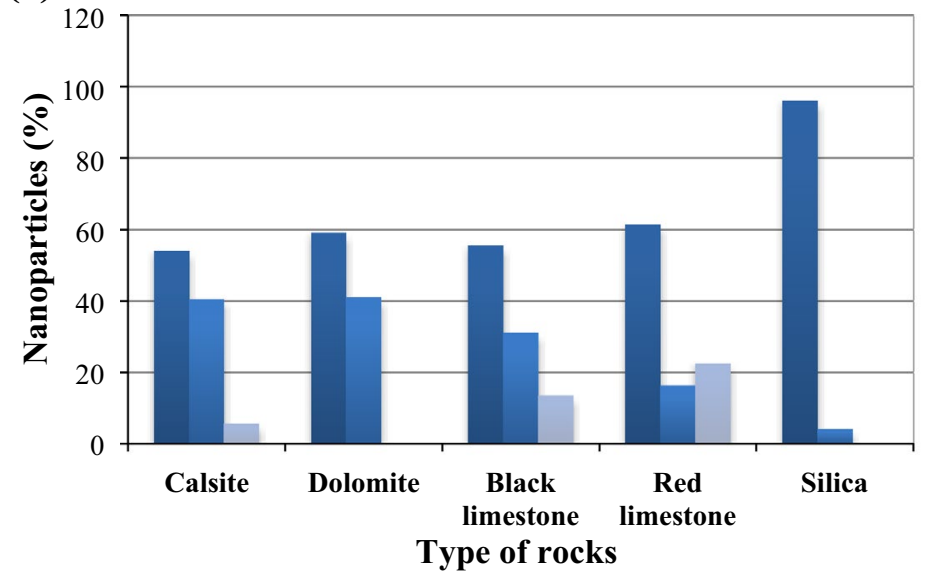

Nanoparticles recovery after flooding

Nanoparticles recovery after post flooding

Nanoparticles Retention

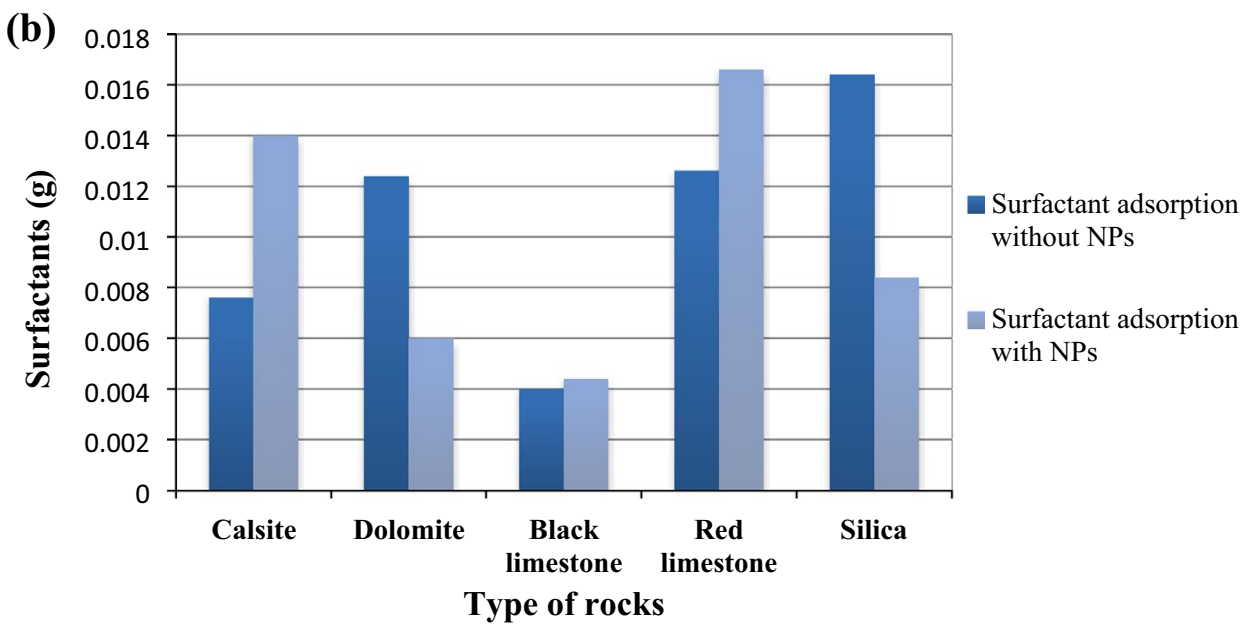

Table $2 \mathrm{TiO}_{2}$ NPs retention and surfactants adsorption in different porous media

\begin{tabular}{|c|c|c|c|c|c|}
\hline Rock type & Calcite & Dolomite & Black limestone & Red limestone & Silica \\
\hline Surfactants adsorption without nanoparticles (mg/g rock) & 1.16 & 0.5 & 1.06 & 1.98 & 1.44 \\
\hline Surfactants adsorption with nanoparticles (mg/g rock) & 1.5 & 0.34 & 1.12 & 2.38 & 0.71 \\
\hline Surfactants adsorption without nanoparticles $\left(\mathrm{mg} / \mathrm{m}^{2}\right.$ rock) & 0.529 & 0.131 & 0.37 & 0.427 & 1.094 \\
\hline Nanoparticle retention (wt $\%)$ & 5.5 & 0 & 13.5 & 22.4 & 0 \\
\hline Nanoparticle retention $\left(\mathrm{mg} / \mathrm{m}^{2}\right.$ rock $)$ & 0.075 & 0 & 0.186 & 0.145 & 0 \\
\hline
\end{tabular}

process also calls "log-jamming". Here the high stability of $\mathrm{TiO}_{2}$ NPs confirms the existence of a strong steric repulsion between particles which prevents their agglomeration during transport. According to Table 1 the surface charge of silica has most negativity and dolomite have the least negativity while both of them have minimum retention of NPs. With attention to the negative zeta potential value of $\mathrm{TiO}_{2} \mathrm{NPs}$, more retention is expected in dolomite rock compared to other rocks. In fact, it seems that the electrostatic attraction between rock surface and NPs would have a minor effect on particle retention because both silica and dolomite rocks, with opposite surface charges, have the minimum amount of NPs deposition. There is the degree of inconsistency between our observation for the effect of surface area and surface charge and other studies which is probably due to two factors: functionalizing of NPs with surfactants blend and surface roughness of rocks. Functionalized surface was covered with a surfactant shell, which reduces the significance of NP's surface charge on NPs retention compared to the bare surface.

Moreover, the SEM images and BET analysis together reveal some detail about the surface roughness and topographical of the rocks. The SEM photo illustrated that the silica and dolomite rocks possessed a crystallized particulate 
Fig. 8 Schematic diagram of main transport mechanisms of NPs deposition in porous media

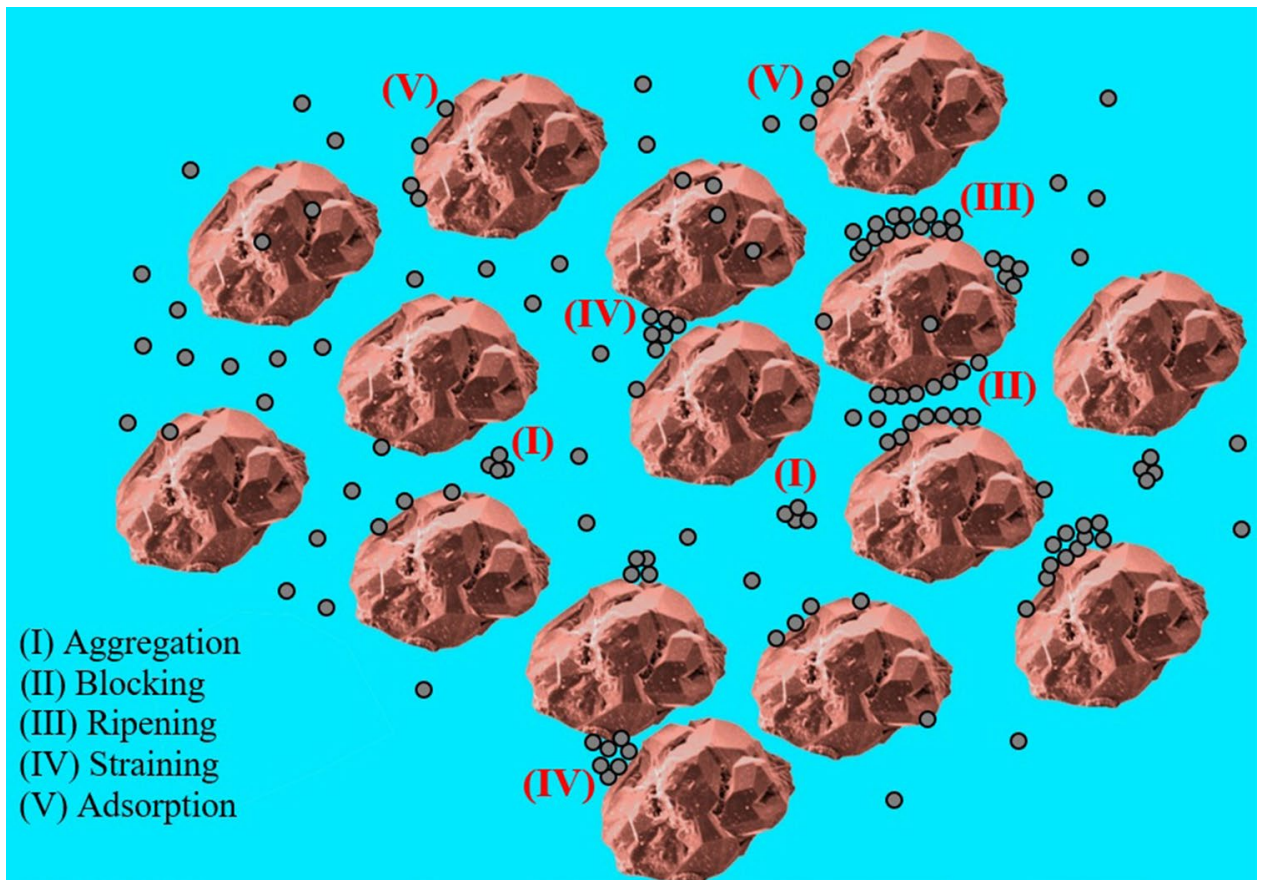

morphology. The SEM images of limestone rocks also illustrate the existence of tiny irregular dents and bumps along with submicrometer particles $(<1 \mu \mathrm{m})$ on the surface of rocks which account for the highest measured BET surface area (Fig. 4). The rougher surface of limestone and calcite rocks caused more retention of NPs (Bayat et al. 2015a, b, c; Jian et al. 2016). SEM and EDEX analyses also were carried out from the surface and bulk of different rock's particles to prove the deposition of NPs. The titanium elemental map of bulk grains indicates a lower density of NPs in silica grains in compared to Black limestone and Calcite (Fig. S7). $\mathrm{TiO}_{2}$ NPs clusters also vividly observed between submicron grains of rock (Fig. S8). Other researchers also stated that the irregular dents and bumps of rock surfaces lead to more NPs to be trapped (Bayat et al. 2015a, b, c; Bradford and Torkzaban 2008).

In absence of NPs and based on the mass of the rock (mg surfactant/g rock), the highest adsorption of surfactants belongs to the red limestone (Table 2); however, based on the surface of the rock ( $\mathrm{mg}$ surfactant $/ \mathrm{m}^{2}$ rock) the silica rock has adsorbed the highest fraction of the surfactants. All evidences confirm that the surfactants intend to adsorb on the rock surface containing $\mathrm{Si}$ and $\mathrm{Al}$ elements. The $\mathrm{Si}$ and $\mathrm{Al}$ elements (source of silica and clay mineral) were found in all rocks structure except the dolomite which has the least surfactants adsorption amount. Basically, the surfactant molecules could adsorb by forming electrostatic or hydrogen bonds between hydrophobic tail or hydrophilic head with the available surface of porous media (Zhang and Somasundaran 2006). High fraction of EA (75 wt \%) and the possibility of formation of hydrogen bond between ethylene oxide group the hydroxyl groups at the rock surface is the most probable mechanism for surfactant adoption (Jian et al. 2016). On the other hand, AAS as an ionic surfactant intent to form an electrostatic bond with the opposite charge mineral in the rock structure (Cui et al. 2012; Somasundaran and Krishnakumar 1997). The surface density of hydroxyl groups for different rocks in this study is ordered as dolomite $<$ limestone $<$ Calcite $<$ silica, because of the abundance of $\mathrm{Si}-\mathrm{O}-\mathrm{H}$ and $\mathrm{Al}-\mathrm{O}-\mathrm{H}$ groups at rock's surface. The main mechanism of surfactants adsorption at the rock surface schematically illustrates in Fig. 9. Therefore, the amount of surfactant adsorption on dolomite and silica rock's surfaces is expected to be relatively lowest and highest, respectively.

In this research, we tried to study the synergistic effect between NPs and surfactant during the flooding process through different porous media. The following conclusions can be extracted from this study:

- The rate of surfactants adsorption strongly is a function of surface charge and chemistry of the porous media. A high fraction of surfactants blend (around $36 \mathrm{wt} . \%$ ) was adsorbed on silica rock that would drastically reduce the efficiency of the practical application of chemical flooding.

- The surfactant adsorption on silica and dolomite rock was reduced to half (around 18 wt.\% for silica) after functionalizing the surfactants with $\mathrm{TiO}_{2}$ NPs. In fact, functionalized NPs have the potential to preserve surfactant molecules from adsorption on the porous walls. 
Fig. 9 Proposed mechanism for ethoxylated surfactant adsorption on rock containing silica

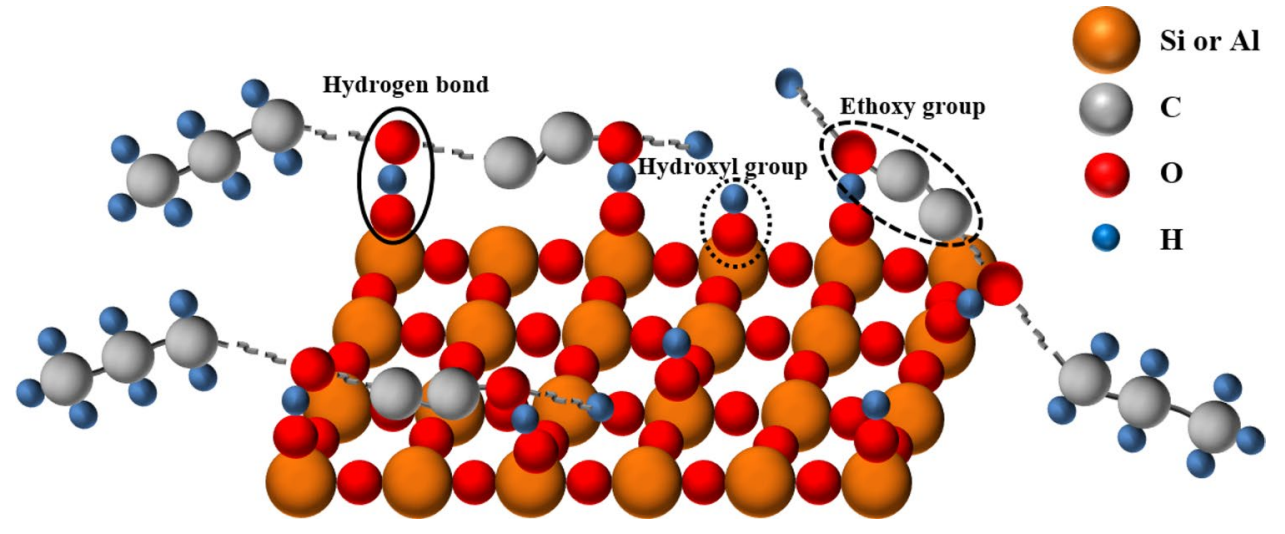

- In spite of the usefulness of NPs for reducing adsorption reduction of surfactant in silica and dolomite rocks, they have an adverse impact in the case of Calcite and limestone rocks. Appling NPs increased the surfactant trapping around 50 and $24 \mathrm{wt} . \%$ in Calcite and red limestone porous media.

- We concluded that the synergistic effect between NPs and surfactant has promising results as long as the NPs deposition in porous media is negligible.

- Making a connection between NPs deposition and physicochemical properties of rock's surface (charge, area, roughness, and wettability) in this study showed that the surface roughness has the most impact on the trapping of NPs. Therefore, the efficiency of functionalized NPs flooding is higher through pore walls with lower microroughness.

\section{Conclusion}

There are several studies dealing with nanoparticle transport through porous media; however, there is still an absence of a comprehensive study to evaluate the potential of NPs as a chemical agent carrier through different types of porous media. Blend of anionic alkylaryl sulfonates and nonionic alcohol ethoxylated surfactant at optimum composition (25-75 wt \%) and salinity (4 wt\%) was used as a stable slug for functionalizing of $\mathrm{TiO}_{2}$ NPs. The core-flooding experiments have been performed in five different types of reservoir rocks. According to the results, the surface roughness of rock had the most impact on the retention of NPs inside the porous media. The SEM photos of limestone grain showed an irregular surface with rare crystal which caused more retention of NPs inside limestone rocks. The adsorption of alkylaryl sulfonates and alcohol ethoxylated blend greatly depend on the mineralogy of rock which was increased at rock surface containing a higher amount of silica and alumina. The hydrogen bonding between the oxygen in the ethoxy groups and the hydroxyl groups of silica suggested as a likely mechanism which is accrued for adsorption of surfactant. Any specific connection between surfactant attachment with wettability or ability of rock was not disclosed in this research. Adsorption of surfactant blend in presence of $\mathrm{TiO}_{2}$ NPs was proportional with retention of NPs inside the column. Therefore, the role of NPs as a carrier for surfactant molecules is outstanding when the transport of NPs through porous media is as much as possible.

Supplementary Information The online version contains supplementary material available at https://doi.org/10.1007/s13204-021-01843-2.

Funding This work was supported by European Research Council Consolidator Grant (Grant number: 648375).

\section{Declarations}

Conflict of interest The authors declare that they have no conflict of interest.

Open Access This article is licensed under a Creative Commons Attribution 4.0 International License, which permits use, sharing, adaptation, distribution and reproduction in any medium or format, as long as you give appropriate credit to the original author(s) and the source, provide a link to the Creative Commons licence, and indicate if changes were made. The images or other third party material in this article are included in the article's Creative Commons licence, unless indicated otherwise in a credit line to the material. If material is not included in the article's Creative Commons licence and your intended use is not permitted by statutory regulation or exceeds the permitted use, you will need to obtain permission directly from the copyright holder. To view a copy of this licence, visit http://creativecommons.org/licenses/by/4.0/.

\section{References}

Agista MN, Andersen PØ, Zhixin Yu (2019) Modelling nanofluid injection in porous media. J Petrol Sci Eng 176:836-849

Arsalan N, Buiting JJ, Nguyen QP (2015) Surface energy and wetting behavior of reservoir rocks. Colloids Surf A Physicochem Eng Asp 467:107-112

Babakhani P, Bridge J, Doong R-a, Phenrat T (2017) Continuum-based models and concepts for the transport of nanoparticles in saturated

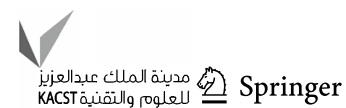


porous media: a state-of-the-science review. Adv Coll Interface Sci 246:75-104

Bayat AE, Junin R, Ghadikolaei FD, Piroozian A (2014) Transport and aggregation of $\mathrm{Al} 2 \mathrm{O} 3$ nanoparticles through saturated limestone under high ionic strength conditions: measurements and mechanisms. J Nanopart Res 16:1-12

Bayat AE, Junin R, Derahman MN, Samad AA (2015a) TiO2 nanoparticle transport and retention through saturated limestone porous media under various ionic strength conditions. Chemosphere 134:7-15

Bayat AE, Junin R, Mohsin R, Hokmabadi M, Shamshirband S (2015b) Influence of clay particles on $\mathrm{Al} 2 \mathrm{O} 3$ and $\mathrm{TiO} 2$ nanoparticles transport and retention through limestone porous media: measurements and mechanisms. J Nanopart Res 17:1-14

Bayat AE, Junin R, Shamshirband S, Chong WT (2015c) Transport and retention of engineered $\mathrm{Al} 2 \mathrm{O} 3, \mathrm{TiO} 2$, and $\mathrm{SiO} 2$ nanoparticles through various sedimentary rocks. Sci Rep 5:14264

Betancur S, Carrasco-Marín F, Pérez-Cadenas AF, Franco CA, Jiménez J, Manrique EJ, Quintero H, Cortés FB (2019) Effect of magnetic iron core-carbon shell nanoparticles in chemical enhanced oil recovery for ultralow interfacial tension region. Energy Fuels 33:4158-4168

Bradford SA, Torkzaban S (2008) Colloid transport and retention in unsaturated porous media: a review of interface-, collector-, and pore-scale processes and models. Vadose Zone J 7:667-681

Cai Y, Xiaoxiao Hu, Li X, Pan Y (2012) Origin of the red colour in a red limestone from the Vispi Quarry section (central Italy): a high-resolution transmission electron microscopy analysis. Cretac Res 38:97-102

Caldelas FM, Murphy M, Huh C, Bryant SL (2011) Factors governing distance of nanoparticle propagation in porous media. In SPE production and operations symposium. Society of Petroleum Engineers

Cui Z-G, Li W, Qi J-J, Wang H-J (2012) Individual and mixed adsorption of alkylcarboxylbetaines and fatty amide ethoxylates at Daqing sandstone/water interface. Colloids Surf A Physicochem Eng Asp 414:180-189

Foroozesh J, Kumar S (2020) Nanoparticles behaviors in porous media: Application to enhanced oil recovery. J Mol Liq 316:113876

Guzman D, Katherine A, Finnegan MP, Banfield JF (2006) Influence of surface potential on aggregation and transport of titania nanoparticles. Environ Sci Technol 40:7688-7693

Haruna MA, Nourafkan E, Zhongliang Hu, Wen D (2019) Improved polymer flooding in harsh environments by free-radical polymerization and the use of nanomaterials. Energy Fuels 33:1637-1648

Hu Z, Gao H, Ramisetti SB, Zhao J, Nourafkan E, Glover PWJ, Wen D (2019) Carbon quantum dots with tracer-like breakthrough ability for reservoir characterization. Sci Total Environ 669:579-589

Jian G, Puerto MC, Wehowsky A, Dong P, Johnston KP, Hirasaki GJ, Biswal SL (2016) Static adsorption of an ethoxylated nonionic surfactant on carbonate minerals. Langmuir 32:10244-10252

Liang Y, Zhou J, Dong Y, Klumpp E, Šimůnek J, Bradford SA (2020) Evidence for the critical role of nanoscale surface roughness on the retention and release of silver nanoparticles in porous media. Environ Pollut 258:113803

Luo D, Wang F, Zhu J, Cao F, Liu Y, Li X, Willson RC, Yang Z, Chu C-W, Ren Z (2016) Nanofluid of graphene-based amphiphilic Janus nanosheets for tertiary or enhanced oil recovery: High performance at low concentration. Proc Natl Acad Sci 113:7711-7716
Medjda R, Khelladi B, Abdelghani CF, Maxime P, Zohra GF (2020) The trapping of colloid particles in porous media: mechanisms and applications, review. J Appl Res Water Wastewater 7:180-188

Nourafkan E, Asachi M, Zhongliang Hu, Gao H, Wen D (2018a) Synthesis of stable nanoparticles at harsh environment using the synergistic effect of surfactants blend. J Ind Eng Chem 64:390-401

Nourafkan E, Zhongliang Hu, Wen D (2018b) Nanoparticle-enabled delivery of surfactants in porous media. J Colloid Interface Sci 519:44-57

Nourafkan E, Haruna MA, Gardy J, Wen D (2019) Improved rheological properties and stability of multiwalled carbon nanotubes/ polymer in harsh environment. J Appl Polym Sci 136:47205

Olayiwola SO, Dejam M (2019) A comprehensive review on interaction of nanoparticles with low salinity water and surfactant for enhanced oil recovery in sandstone and carbonate reservoirs. Fuel 241:1045-1057

De Avila NL, Jocasta, De Araujo LLGC, Drexler S, de Almeida Rodrigues J, Nascimento RSV (2016) Polystyrene nanoparticles as surfactant carriers for enhanced oil recovery. J Appl Polym Sci 133(32)

Qin Y, Wen Z, Zhang W, Chai J, Liu D, Shengyu Wu (2020) Different roles of silica nanoparticles played in virus transport in saturated and unsaturated porous media. Environ Pollut 259:113861

Schramm LL, Mannhardt K, Novosad JJ (1991) Electrokinetic properties of reservoir rock particles. Colloids Surf 55:309-331

Somasundaran P, Krishnakumar S (1997) Adsorption of surfactants and polymers at the solid-liquid interface. Colloids Surf A Physicochem Eng Asp 123:491-513

Venancio JC, Carvalho RS, Nascimento V, Pérez-Gramatges A (2020) Colloidal stability and dynamic adsorption behavior of nanofluids containing alkyl-modified silica nanoparticles and anionic surfactant. J Mol Liq 308:113079

Vijapurapu CS, Rao DN, Kun L (2002) The effect of rock surface characteristics on reservoir wettability. In SPE/DOE Improved Oil Recovery Symposium. Society of Petroleum Engineers

Wenzel RN (1936) Resistance of solid surfaces to wetting by water. Ind Eng Chem 28:988-994

Wu Y, Chen W, Dai C, Huang Y, Li H, Zhao M, He L, Jiao B (2017) Reducing surfactant adsorption on rock by silica nanoparticles for enhanced oil recovery. J Petrol Sci Eng 153:283-287

Yekeen N, Manan MA, Idris AK, Samin AM, Risal AR (2017) Experimental investigation of minimization in surfactant adsorption and improvement in surfactant-foam stability in presence of silicon dioxide and aluminum oxide nanoparticles. J Petrol Sci Eng 159:115-134

Yekeen N, Padmanabhan E, Idris AK, Ibad SM (2019) Surfactant adsorption behaviors onto shale from Malaysian formations: Influence of silicon dioxide nanoparticles, surfactant type, temperature, salinity and shale lithology. J Petrol Sci Eng 179:841-854

Zhang R, Somasundaran P (2006) Advances in adsorption of surfactants and their mixtures at solid/solution interfaces. Adv Coll Interface Sci 123:213-229

Publisher's Note Springer Nature remains neutral with regard to jurisdictional claims in published maps and institutional affiliations. 